\title{
Peritoneal Encapsulation With Incarcerated Meckel Diverticulum Contributing to Small Bowel Obstruction
}

\author{
Keenan J. Robbins, BS, ${ }^{1}$ Hannah Z. Kooperkamp, MD, ${ }^{2}$ Ralph L. Corsetti, MD $^{1,2}$ \\ ${ }^{1}$ The University of Queensland Faculty of Medicine, Ochsner Clinical School, New Orleans, LA ${ }^{2}$ Department of Surgery, Ochsner Clinic \\ Foundation, New Orleans, LA
}

\begin{abstract}
Background: Meckel diverticulum is the most common congenital gastrointestinal malformation, but it is rarely symptomatic. Peritoneal encapsulation is a rare abnormality of embryonic development and may present with obstruction, although it too is usually asymptomatic.

Case Report: We report the case of an 82-year-old male who presented with symptoms and imaging findings consistent with small bowel obstruction. During laparotomy, a peritoneal encapsulation was observed containing the majority of the small bowel. A Meckel diverticulum was discovered tethered to the small bowel mesentery by a mesodiverticular band, preventing egress from the accessory peritoneal membrane and appearing to be the cause of the obstruction.

Conclusion: This patient had 3 rare findings-peritoneal encapsulation, Meckel diverticulum, and a mesodiverticular bandcontributing to cause an acute small bowel obstruction. The presence of all of these features in one patient is extremely rare, and we believe this is the first description of such a phenomenon.
\end{abstract}

Keywords: Hernia-abdominal, intestinal obstruction, Meckel diverticulum

Address correspondence to Ralph L. Corsetti, MD, Department of Surgery, Ochsner Clinic Foundation, 1514 Jefferson Hwy., New Orleans, LA 70121. Tel: (504) 842-2444. Email: rcorsetti@ochsner.org

\section{INTRODUCTION}

A Meckel diverticulum is a vestige of the fetal omphalomesenteric duct and is the most common congenital gastrointestinal (GI) malformation. It is thought to occur in $2 \%-4 \%$ of the population ${ }^{1}$ and is rarely symptomatic. Most cases of symptomatic Meckel diverticula occur in children, and Gl bleeding is the most common manifestation. ${ }^{2}$ Bleeding can also occur in adults, but the most common presentation is obstruction, followed by ulceration, diverticulitis, or perforation. ${ }^{3}$ Preoperative diagnosis of a Meckel diverticulum is difficult, and its existence is usually discovered incidentally during surgery for another indication. ${ }^{4}$

Peritoneal encapsulation is an exceptionally rare phenomenon in which an anomaly during the physiologic midgut herniation stage of embryonic development results in an accessory peritoneal membrane surrounding some or all of the small bowel. ${ }^{5}$ Although usually asymptomatic, peritoneal encapsulation can present with recurrent episodes of colicky abdominal pain or acute small bowel obstruction. Like a Meckel diverticulum, peritoneal encapsulation is difficult to appreciate on imaging and is most often diagnosed unexpectedly in the operating room.

We report what we believe to be the first documented case of a Meckel diverticulum incarcerated within a peritoneal encapsulation and causing acute small bowel obstruction.

\section{CASE REPORT}

An 82-year-old male presented to the emergency department with 24 hours of diffuse abdominal pain accompanied by nausea and anorexia. His last bowel movement was the afternoon of presentation. His medical history was significant for 2 episodes of painless rectal bleeding more than 15 years prior that were attributed to diverticulitis at the time. The patient also reported a postoperative ileus after a bilateral total knee arthroplasty in 2003. He had no history of abdominal operations. While his last colonoscopy had been performed more than 10 years prior, the patient had no history of abnormal colonoscopies. He was afebrile and had a blood pressure of $174 / 78 \mathrm{mmHg}$, but vital signs were otherwise normal. His examination was remarkable for a firm, distended abdomen that was diffusely tender to palpation with focally increased tenderness in the left upper and lower quadrants. No rigidity, rebound tenderness, or appreciable masses were noted. An easily reducible incidental umbilical hernia was noted that had not bothered the patient. Computed tomography (CT) showed dilation of the majority of the small bowel and decompressed terminal ileum without an identifiable transition point. The scan showed scattered diverticula but no evidence of diverticulosis.

Given the patient's age and absence of prior abdominal surgeries, the presentation and imaging findings were concerning for an internal hernia or malignancy as the 


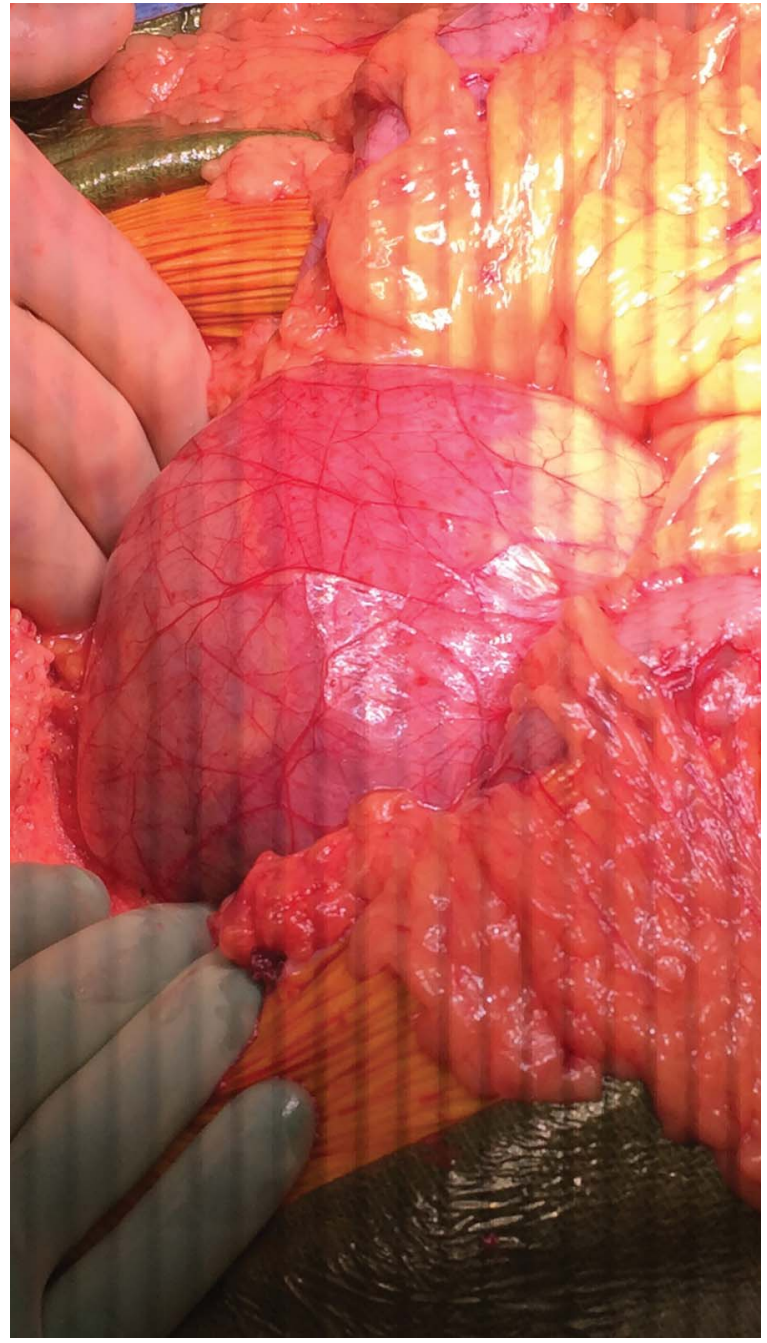

Figure 1. A thin, translucent membrane encased the small bowel from the ligament of Treitz to the terminal ileum.

underlying etiology of the small bowel obstruction. The patient was taken to the operating room for an exploratory laparotomy.

Inspection of the peritoneal cavity showed no evidence of intestinal malrotation, carcinomatosis, or infection. However, a large, well-vascularized, thin, translucent membrane encased the small bowel from the ligament of Treitz to the terminal ileum (Figure 1). The membrane was incised, and a segment was resected and sent to pathology despite its overall benign appearance. The bowel was then run from the ligament of Treitz distally, decompressing the bowel via an 18 French nasogastric tube in the process. Near the terminal ileum was a clear transition point with dilated proximal bowel and normal bowel distally. On the antimesenteric side of this transition point, we discovered a Meckel diverticulum, measuring $3.1 \times 2.2 \mathrm{~cm}$ (Figure 2). The distal aspect of the diverticulum was tethered into the small bowel mesentery via a mesodiverticular band, potentially serving as a point of fixation leading to kinking of the bowel or preventing egress from the peritoneal encapsulation. Upon palpation, the Meckel diverticulum was noted to be abnormally

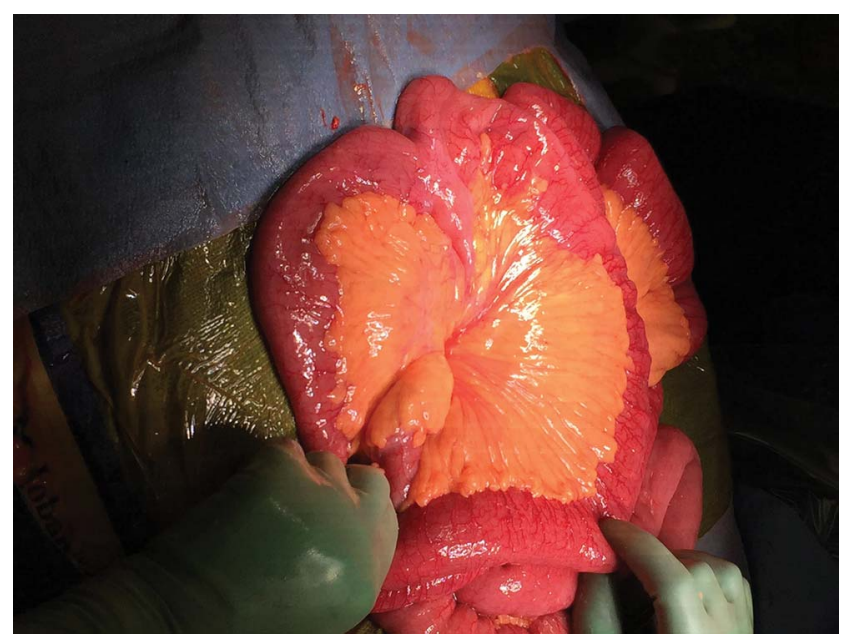

Figure 2. A Meckel diverticulum was observed in the distal ileum. The apex of the diverticulum was tethered to the small bowel mesentery by a mesodiverticular band.

thickened, possibly consistent with ectopic gastric or pancreatic mucosa in the lumen of the diverticulum. The pathology report did not mention the presence of ectopic tissue but noted ischemic and reactive mucosal changes. Because we thought the Meckel diverticulum contributed to the obstructive process and because of diverticular wall thickening in the setting of a history of Gl bleeding, we elected to resect the diverticulum. Short margins of ileum on either side of the diverticulum were included in the resection, and the bowel was reanastomosed using a stapled side-to-side technique. The bowel was then returned to the abdomen, the incidental umbilical hernia was repaired, the fascia was closed with running looped \#1 PDS suture, and the subcutaneous tissue was irrigated thoroughly before skin closure with staples.

Bowel function returned on postoperative day 4, the patient's diet was advanced, and he was discharged home

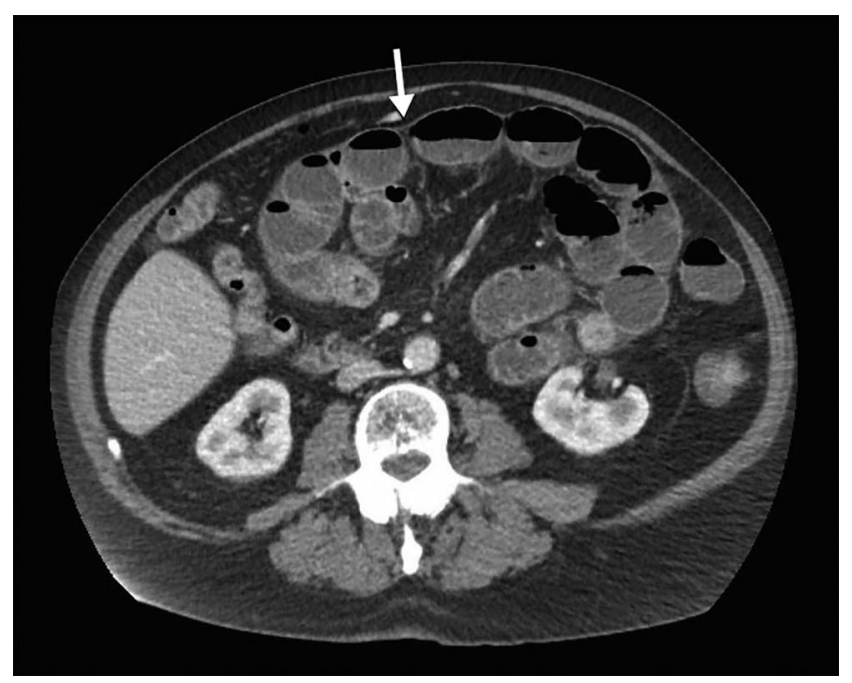

Figure 3. Computed tomography shows the small bowel abnormally localized in the upper left portion of the abdomen. An appreciable thin membrane, denoted by an arrow, surrounds the small bowel, representing the peritoneal encapsulation. 


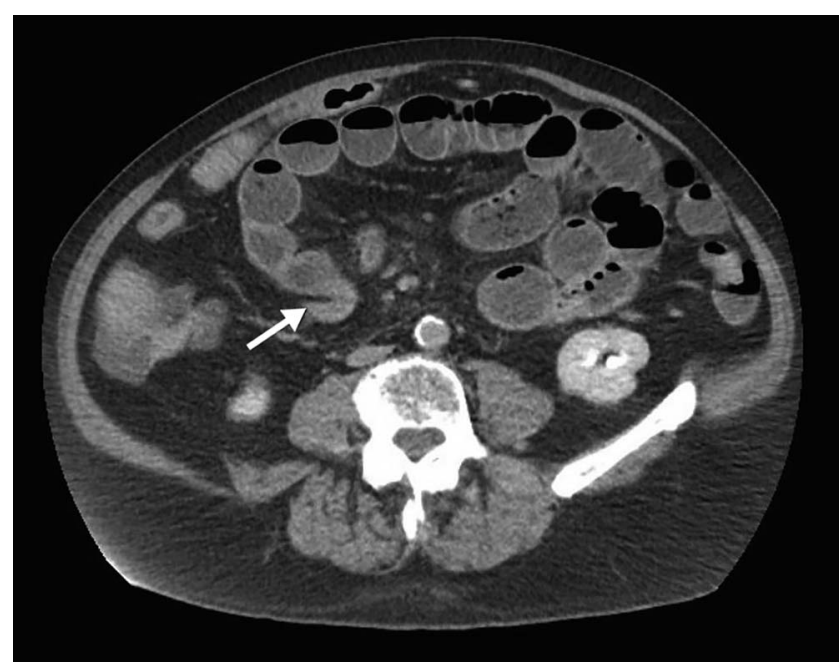

Figure 4. The distal ileum makes a sharp turn, and an appreciable transition point is at what appears to be the point of egress from the accessory peritoneal membrane. The decompressed distal bowel is indicated by an arrow.

on postoperative day 8. The patient attended all followup appointments, and his convalescence had no significant deviations from the normal course. He continues to do well without recurrence of symptoms.

\section{DISCUSSION}

Isolated case reports have suggested the inclusion of peritoneal encapsulation in the differential for acute bowel obstruction in patients without a history of abdominal surgery. ${ }^{6}$ Other case reports have suggested characteristic imaging findings to aid in preoperative identification of this anomaly. ${ }^{7}$ Reviewing the CT with the benefit of hindsight, an appreciable thin membrane does appear to surround an unusually localized collection of small bowel (Figure 3). This finding was commented upon preoperatively, but the significance was unclear. The collection of bowel appears to displace the middle colic vein, forcing it into an unusually tortuous course. A clearly demarcated plane between the collection of bowel and adjacent colon and other structures is suggestive of the small bowel residing in an accessory sac. At what appears to be the point of egress from this sac, the ileum turns abruptly and is decompressed distally (Figure 4). This area may represent the point of obstruc- tion and corresponds with where we discovered the Meckel diverticulum intraoperatively.

The rarity of peritoneal encapsulation, the rarity of Meckel diverticulum, and the typically quiescent existence of both entities make it extremely unlikely for both to not only be present in the same individual but to contribute to what was likely an interdependent pathology. Since the first description of peritoneal encapsulation in 1868, only 30 instances have been reported in the medical literature. ${ }^{8}$ As none of the reported cases we had access to described peritoneal encapsulation with an associated Meckel diverticulum causing acute small bowel obstruction, we believe this case report to be the first description of this phenomenon.

\section{CONCLUSION}

The infrequency of symptomatic peritoneal encapsulation is such that it will likely continue to be an incidental finding during laparotomy under the presumption of other more common causes of acute abdomen.

\section{ACKNOWLEDGMENTS}

The authors have no financial or proprietary interest in the subject matter of this article.

\section{REFERENCES}

1. Elsayes KM, Menias CO, Harvin HJ, Francis IR. Imaging manifestations of Meckel's diverticulum. AJR Am J Roentgenol. 2007 Jul;189(1):81-88.

2. Park JJ, Wolff BG, Tollefson MK, Walsh EE, Larson DR. Meckel diverticulum: the Mayo Clinic experience with 1476 patients (1950-2002). Ann Surg. 2005 Mar;241(3):529-533.

3. Sagar J, Kumar V, Shah DK. Meckel's diverticulum: a systematic review. J R Soc Med. 2006 Oct;99(10):501-505.

4. Sharples AJ. Meckel diverticulum causing small bowel obstruction. BMJ Case Rep. 2010;2010. doi: 10.1136/bcr.06.2009.1970.

5. Naraynsingh V, Maharaj D, Singh M, Ramdass MJ. Peritoneal encapsulation: a preoperative diagnosis is possible. Postgrad Med J. 2001 Nov;77(913):725-726.

6. Griffith D, Boal M, Rogers T. Peritoneal encapsulation; a rare cause of bowel obstruction. Ann R Coll Surg Engl. 2017 Jan;99(1):e11-e12. doi: 10.1308/rcsann.2016.0264.

7. Casas JD, Mariscal A, Martínez N. Peritoneal encapsulation: CT appearance. AJR Am J Roentgenol. 1998 Oct;171(4):1017-1019.

8. Arumugam PK, Dalal AK. Peritoneal encapsulation - an unexpected cause of acute intestinal obstruction. J Visc Surg. 2017 Sep;154(4):303-305. doi: 10.1016/j.jviscsurg.2017.06.001.

This article meets the Accreditation Council for Graduate Medical Education and the American Board of Medical Specialties Maintenance of Certification competencies for Patient Care and Medical Knowledge. 